\title{
Role of HER-2 activity in the regulation of malignant meningioma cell proliferation and motility
}

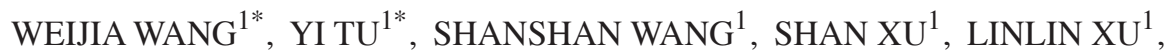 \\ YIFENG XIONG $^{1}$, JINHONG MEI ${ }^{1}$ and CHUNLIANG WANG ${ }^{2}$ \\ Departments of ${ }^{1}$ Pathology and ${ }^{2}$ Neurosurgery, The First Affiliated Hospital of Nanchang University, \\ Nanchang, Jiangxi 330006, P.R. China
}

Received August 5, 2014; Accepted April 24, 2015

DOI: $10.3892 / \mathrm{mmr} .2015 .3805$

\begin{abstract}
Meningiomas are common types of intracranial tumor. Invasive and malignant meningiomas present a significant therapeutic challenge due to high rates of recurrence and invasion. Understanding the molecular mechanism of invasion may assist in designing novel therapeutic approaches and improving patient survival rates. The HER-2 gene has been demonstrated to be a useful predictor of tumor aggression, which promotes the survival and growth of cancer cells through the mitogen-activated protein kinase and/or phosphatidylinositol 3-kinase (PI3K)/AKT pathway. Until now, few studies have investigated the associateion between meningiomas and the expression of HER-2, and the significance of HER-2 in meningiomas remains to be elucidated. The present study aimed to investigate the effects of the HER-2 gene on the biological behaviors of human malignant meningioma cells. The results demonstrated that downregulation of the expression of HER-2 by small interfering RNA in human meningioma cells significantly inhibited cell motility and proliferation, led to cell cycle arrest at the G0/G1-phase and increased early apoptosis. By contrast, the overexpression of HER-2 group resulted in meningioma cell invasion, migration and proliferation being significantly enhanced, cell cycle was promoted at the G1/S-phase and early apoptosis was decreased. Accordingly, the inhibition of HER-2 also prevented the protein expression of PI3K and phosphorylated AKT. The results demonstrated
\end{abstract}

Correspondence to: Professor Chunliang Wang, Department of Neurosurgery, The First Affiliated Hospital of Nanchang University, 17 Yongwai Zheng Road, Nanchang, Jiangxi 330006, P.R. China E-mail: wang330006@126.com

Professor Jinhong Mei, Department of Pathology, The First Affiliated Hospital of Nanchang University, 17 Yongwai Zheng Road, Nanchang, Jiangxi 330006, P.R. China

E-mail: mjhdoctor@126.com

\section{*Contributed equally}

Key words: human malignant meningioma, HER-2, proliferation, motility that regulation of the HER-2 gene can affect the proliferation, apoptosis, invasion and metastasis abilities of human meningioma cells in vitro. Furthermore, PI3K/AKT may contribute to the carcinogenesis and development of human meningiomas in combination with HER-2.

\section{Introduction}

Meningiomas are the second most common type of tumor of the primary central nervous system and accounts for $24-30 \%$ of all reported brain tumors, with malignant meningiomas (WHO grade III) accounting for 1-3\% of meningiomas (1-3). Invasive and malignant meningiomas present a significant therapeutic challenge due to high rates of recurrence and invasion into the surrounding bone, brain, neural and soft tissues $(4,5)$. Therefore, understanding the molecular mechanism of invasion may assist in designing novel therapeutic approaches to reduce the requirement for repeat surgery, decrease morbidity rates and improve patient survival rates.

The HER-2 gene is a type of proto-oncogene, also termed CerbB-2 or neu, is located on chromosome 17, encodes a $185 \mathrm{kDa}$ transmembrane receptor tyrosine kinase (RTK), and is a member of the epidermal growth factor receptor (EGFR) family, which contains four homologous members: EGFR/HER-1, HER-2, HER-3 and HER-4 (6). HER-2 receptors exhibit functional redundancy with overlapping signaling pathways. HER-2 is the preferred heterodimerization partner of all EGFR proteins, and is important in the lateral transmission of signals between other HER receptors (7). HER receptor pathway-associated proteins are important in normal cells, as well as in cancer cells. Activation of $185 \mathrm{kDa}$ RTKs, which are transmembrane receptors with an intrinsic ability to phosphorylate tyrosine residues in their cytoplasmic domains, including phosphatidylinositol 3-kinase (PI3k) and AKT, results in activation of nuclear transcription factors that induce cell growth and inhibit apoptosis (8). Therefore, HER-2 signaling has been reported to induce several phenotypic changes associated with more aggressive disease, including increased cell motility, cell proliferation and/or invasive ability (9).

Since the association between HER-2 gene amplification and disease prognosis was demonstrated $(10,11)$, overexpression of the gene has been observed in a variety of primary human carcinoma, including breast cancer $(12,13)$, gastric 
cancer $(14,15)$, gastroesophageal adenocarcinomas (16) and mucinous epithelial ovarian cancer (17). Initially, HER-2 was found to be upregulated in meningioma (18-21). Subsequently, several studies have provided evidence that Her-2 is important in meningiomas $(19,20)$. However, the association and mechanism between meningiomas and the expression of HER-2 remain to be fully elucidated.

In the present study, the possible role of HER-2 in cell proliferation, apoptosis and invasion of the IOMM-Lee human malignant meningioma cell line was investigated. The results may provide clues for further investigation of the mechanisms underlying the carcinogenesis of malignant meningioma and provide a candidate gene for meningioma therapy.

\section{Materials and methods}

Cell lines and cell culture. The IOMM-Lee human meningioma cell line was provided by Dr Jensen and Dr Gillespie of the University of Utah (Salt Lake City, USA). The cell line was routinely cultured in Dulbecco's modified Eagle's medium (DMEM; GE Healthcare, Logan, UT, USA), supplemented with $10 \%$ fetal bovine serum (FBS; Gibco-BRL, Carlsbad. CA, USA), $100 \mathrm{U} / \mathrm{ml}$ streptomycin and $100 \mathrm{U} / \mathrm{ml}$ penicillin (Beijing Solarbio Science \& Technology Co., Ltd., Beijing, China), in a humidified $37^{\circ} \mathrm{C}$ incubator containing $5 \% \mathrm{CO}_{2}$.

Plasmids and transfection. Fragments of the short hairpin (sh) HER-2 (HER-2-sh) and HER-2-overexpression sequence were obtained through NCBI references from HanBio (Shanghai, China) and the NM_004448 GenBank HER2 NCBI reference sequence, respectively, then the HER-2-sh and HER-2-overexpression lentiviral vectors were constructed. Nonsense sequence lentiviral vectors (NC-sh and NC-overexpression) were used as negative controls. The HER-2-sh lentiviral vectors were synthesized by HanBio (Shanghai, China) and the HER-2-overxepression lentiviral vectors were synthesized by GeneChem (Shanghai, China). The IOMM-Lee cells were transfected with each group and puromycin was used to screen for stable cell lines. Cells at $30-50 \%$ confluence were transfected with the lentivirus at a multiplicity of infection of 10 with enhanced infection solution (7 $\mu \mathrm{g} / \mathrm{ml}$; GeneChem) for $8 \mathrm{~h}$ and washed with fresh medium. Stably transfected cells were selected with $2 \mu \mathrm{g} / \mathrm{ml}$ puromycin (Sigma-Aldrich, St. Louis, MO, USA) for 2 weeks. Stable transformants were identified by fluorescence microscopy, reverse transcription-quantitative polymerase chain reaction (RT-qPCR) and western blot analysis.

RT-qPCR analysis. Total RNAs were extracted from the transfected IOMM-Lee cells using TRIzol reagent (Invitrogen Life Technologies, Carlsbad, CA, USA). The RNA quality and concentration were analyzed by measuring the absorbance at 260 and $280 \mathrm{~nm}$ with the spectrometer (759S; Shanghai Lengguang Technology Co., Ltd., Shanghai, China), and the A260/A280 ratios were between 1.8 and 2.0. For single-stranded cDNA synthesis, the RT reaction was performed using a Revertaid First Strand cDNA synthesis kit (Transgen, Beijing, China).qPCR was then performed with $1 \mu \mathrm{g}$ RNA and $1 \mu \mathrm{l}$ of the following primers from Invitrogen Life Technologies: HER-2, forward 5'-CGGACGCCTGATGGGTTAAT-3' (120 bp) and reverse 5'-ACAGCAAAGGTTCTACCCCG-3' and GAPDH, forward 5'-CAGGGCTGCTTTTAACTCTGGT-3' (203 bp) and reverse 5'-GATTTTGGAGGGATCTCGCT-3'. The qPCR procedure conducted in the ABI PRISM 7500 system (Applied Biosystems, Waltham, MA, USA) was as follows: denaturing at $95^{\circ} \mathrm{C}$ for $2 \mathrm{~min}$ and 40 cycles of annealing at $95^{\circ} \mathrm{C}$ for $15 \mathrm{sec}$ and extension at $58^{\circ} \mathrm{C}$ for $30 \mathrm{sec}$.

Western blot analysis. The stable cells, which in the exponential growth phase, were seeded into 6-well plates and were allowed to grow until $80-90 \%$ confluence, following which they were lysed in lysis buffer (Beyotime Institute of Biotechnology, Beijing, China) on ice. The cells were harvested, washed twice with $1 \mathrm{X}$ phosphate-buffered saline (PBS) and lysed in $100 \mu \mathrm{l}$ radioimmunoprecipitation assay lysis buffer (Vazyme Biotech (Nanjing) Co., Ltd., Nanjing,China). Protein concentrations were determined using a bicinchoninic acid kit (Vazyme Biotech (Nanjing) Co., Ltd.). The proteins were separated using 10\% SDS-PAGE and were then blotted onto nitrocellulose membranes by wet electroblotting at a constant current $200 \mathrm{~mA}$ for $2 \mathrm{~h}$. The membranes were blocked with $5 \%$ non-fat milk powder at room temperature for $1 \mathrm{~h}$ and incubated overnight with the following primary antibodies: Monoclonal mouse HER-2 (3B5; 1:500; Abcam, Cambridge, MA, USA), polyclonal rabbit PI3K (C73F8; 1:1,000; Cell Signaling Technology, Inc., Danvers, MA, USA), polyclonal rabbit phosphorylated (p)-PI3K (19H8; 1:1,000; Cell Signaling Technology, Inc.), polyclonal rabbit AKT (193H12; 1:1,000; Cell Signaling Technology, Inc.), polyclonal rabbit p-AKT (D5G4; 1:1,000; Cell Signaling Technology, Inc.) and monoclonal mouse $\beta$-actin (T0022; 1:5,000; Cell Signaling Technology, Inc.) at $4^{\circ} \mathrm{C}$. Following incubation, the membrane was rinsed with Tris-buffered saline with Tween 20 (TBST) for $15 \mathrm{~min}$ three times, and incubated with secondary antibody. The membrane was agitated for $1 \mathrm{~h}$ at room temperature, washed again in TBST, and were developed using an ECL Plus western blotting detection system (Bio-Rad Laboratories, Inc., Hercules, CA, USA).

MTT analysis. To investigate the proliferation ability of the four cell groups, the present study performed an MTT assay. The stable cells, in the exponential growth phase, were trypinized, centrifuged at $400 \mathrm{x}$ g for $5 \mathrm{~min}$ at room temperature, resuspend in complete medium (10\% FBS) and were counted. The cells were then seeded into five 96-well plates $\left(1 \times 10^{3}\right.$ cells/well), with five parallel wells for each cell group. The medium was replaced with $120 \mu 1$ MTT solution, containing $100 \mu \mathrm{l}$ medium and $20 \mu \mathrm{l}$ MTT (Beijing Solarbio Science \& Technology Co., Ltd.), at different time-points (24, 48, 72, 96 and $120 \mathrm{~h}$ ). After $4 \mathrm{~h}$, the medium was aspirated and $150 \mu \mathrm{l}$ DMSO was added to each well, then the plate was agitated for $45 \mathrm{sec}$ at $27^{\circ} \mathrm{C}$. The optical density value at a wavelength of $490 \mathrm{~nm}$ was determined using a Multiskan FC Microplate photometer (Thermo Fisher Scientific, Waltham, MA, USA).

Colony formation assay. The cells ( $1 \times 10^{3}$ cells) were plated into $6 \mathrm{~cm}$ plates at $37^{\circ} \mathrm{C}$. After 2 weeks, the cells were fixed with methanol and stained using $0.1 \%$ crystal violet (Beijing Solarbio Science \& Technology Co., Ltd.). The number of 
colonies, defined as $\geq 50$ cells/colony, was then counted using a BX61 microscope (Olympus, Tokyo, Japan). The experiments were performed in triplicate.

EdU labeling assay. The stable cells, in the exponential growth phase, were seeded into 6 -well plates and were allowed to grow until $50 \%$ confluence at $37^{\circ} \mathrm{C}$. EdU (Guangzhou RiboBio Co., Ltd., Guangzhou, China) was then added to the culture media in concentrations of $50 \mu \mathrm{mol}$ for $2 \mathrm{~h}$. Following EdU labeling, the cells were washed twice with PBS and fixed with $4.0 \%$ formaldehyde for $30 \mathrm{~min}$ at room temperature. The cells were then fixed in $0.2 \%$ Triton X-100 (Beyotime Institute of Biotechnology) for $10 \mathrm{~min}$ at $37^{\circ} \mathrm{C}$. The EdU-stained cells were immunostained using the Cell-Light ${ }^{\mathrm{TM}}$ EdU Apollo ${ }^{\circledR} 643$ In Vitro Imaging kit (Guangzhou RiboBio Co., Ltd.) and, following washing twice with PBS, the cells were counterstained using $1.5 \mu \mathrm{g} / \mathrm{ml}$ Hoechst 33342 (Guangzhou RiboBio Co., Ltd.), mounted in standard mounting media and imaged using confocal microscopy (LSM700; Zeiss, Jena, Germany). The EdU stain was stable indefinitely at $\leq 4^{\circ} \mathrm{C}$.

Cell invasion and migration analysis. Cell invasion was analyzed using a Transwell chamber containing 24-well plates with an $8 \mu \mathrm{m}$ membrane. The cells were added to the upper chambers, respectively, in each group, and the bottom of the chamber was coated with $1 \mathrm{mg} / \mathrm{ml}$ Matrigel for invasion assays. The cells $\left(3 \times 10^{4}\right)$ were seeded onto the Matrigel-coated Transwell chambe. Cell migration analysis was performed using a wound healing assay, in which stable cells in the exponential growth phase were trypsinized and seeded onto a 6-well plate at $1 \times 10^{6}$ cells/well, with two wells for each cell group (HER-2-sh-NC, HER-2-sh, HER-2-over-NC and HER-2-over). Following incubation overnight at $37^{\circ} \mathrm{C}$, two parallel wounds of $\sim 400 \mu \mathrm{m}$ were made using a $10 \mu \mathrm{l}$ pipette tip. Following rinsing with PBS three times, the cells were plated in FBS-free DMEM medium supplement with penicillin/streptomycin ( $2 \mathrm{ml} /$ well). Images of the cells were captured at 0 and $24 \mathrm{~h}$, and the distance migrated was measured. The cell migration rate was obtained by counting three fields per area, with results presented as the average of six independent experiments performed over multiple days.

Cell cycle and apoptotic assays using flow cytometry. The stable cells, in the logarithmic growth phase, were seeded into a 6 -well plate $\left(2 \times 10^{5}\right.$ cells/well), and were trypsinized and resuspended the following day at $37^{\circ} \mathrm{C}$ overnight. The cells were washed with PBS twice at $400 \mathrm{x}$ g for $5 \mathrm{~min}$. For cell cycle analysis, propidium iodide was attributable to the cell cycle and the distribution of cells was analyzed using flow cytometry (FC500; Beckman Coulter, Inc., Brea, CA, USA). For analysis of apoptosis, an Annexin-V-PE/7-AAD apoptosis detection kit (Nanjing KeyGen Biotech. Co., Ltd., Nanjing, China) was used, according to the manufacturer's instructions. Apoptosis was analyzed using flow cytometry. The cells undergoing apoptosis were annexin V-PE-positive and 7-AAD-negative.

Statistical analysis. Statistical analysis was performed using SPSS 18.0 software (SPSS Inc., Chicago, IL, USA). The data are presented as the mean \pm standard deviation. Statistical
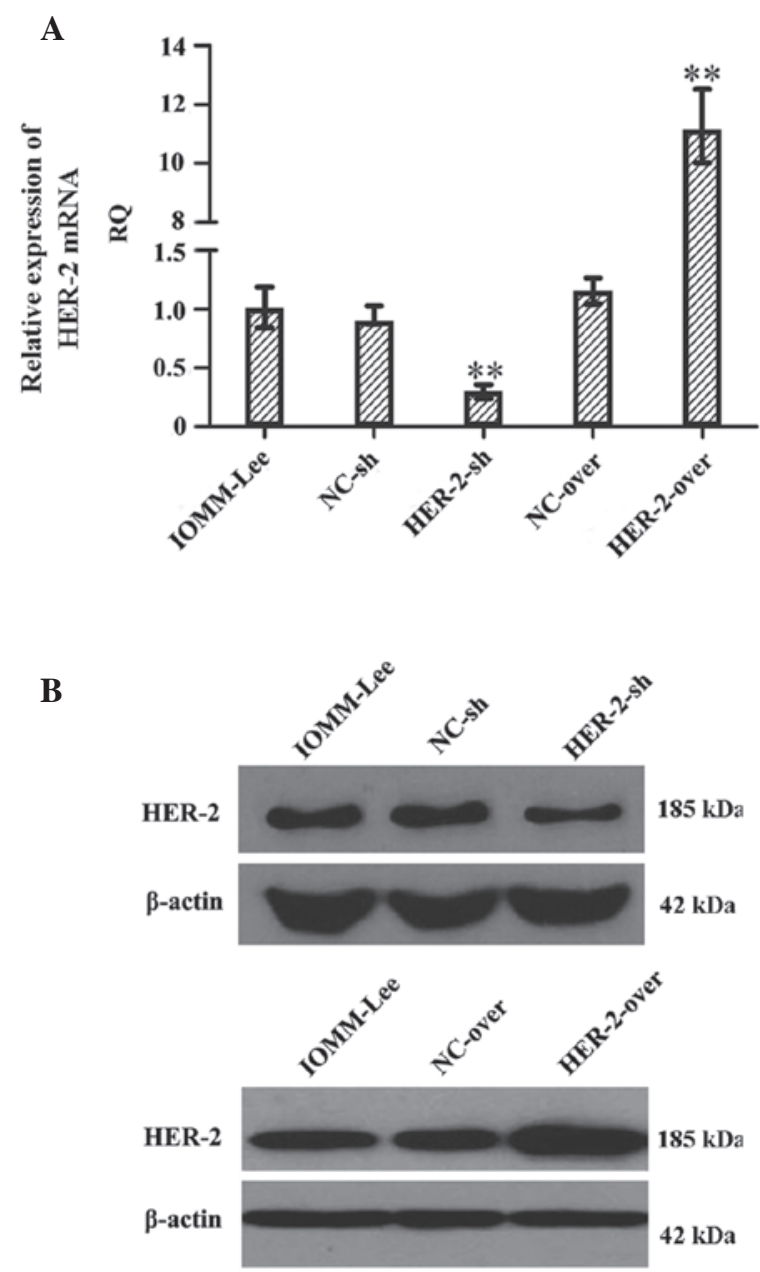

Figure 1. Effect of the HER-2 gene on the expression of HER-2 in IOMM-Lee cells. (A) mRNA levels of HER-2 in the IOMM-Lee malignant meningioma cells. Compared with the NC control, the mRNA expression of HER-2 in the IOMM-Lee cells transfected with HER-2-sh was decreased, and the expression of HER-2 in the IOMM-Lee cells transfected with HER-2-over was significantly increased $\left({ }^{* *} \mathrm{P}<0.01\right)$. (B) Protein expression levels of HER-2 in IOMM-Lee malignant meningioma cells transfected with HER-2-sh, HER-2-over, NC-sh or NC-over, determined using western blot analysis. The data are expressed as the mean standard deviation from three independent experiments. NC, negative control; sh, short hairpin; over, overexpression.

analyses were performed using analysis of variance or Student's t-test. $\mathrm{P} \leq 0.05$ was considered to indicate a statistically significant difference.

\section{Results}

Expression of HER-2 in human meningioma cell lines. In order to investigate the function of HER-2 in the IOMM-Lee cells, the present study examined the expression of HER-2 in the IOMM-Lee cell lines using RT-qPCR following transfection for $96 \mathrm{~h}$ (Fig. 1A). The expression of HER-2 in the IOMM-Lee cells transfected with HER-2-sh decreased $67.2 \%$, compared with the negative control cells or the cells transfected with NC-sh $(\mathrm{P}<0.01)$. The expression of HER-2 in the HER-2-overexpression IOMM-Lee cells was significantly increased (8.7-fold), compared with the mock or NC cells 
A

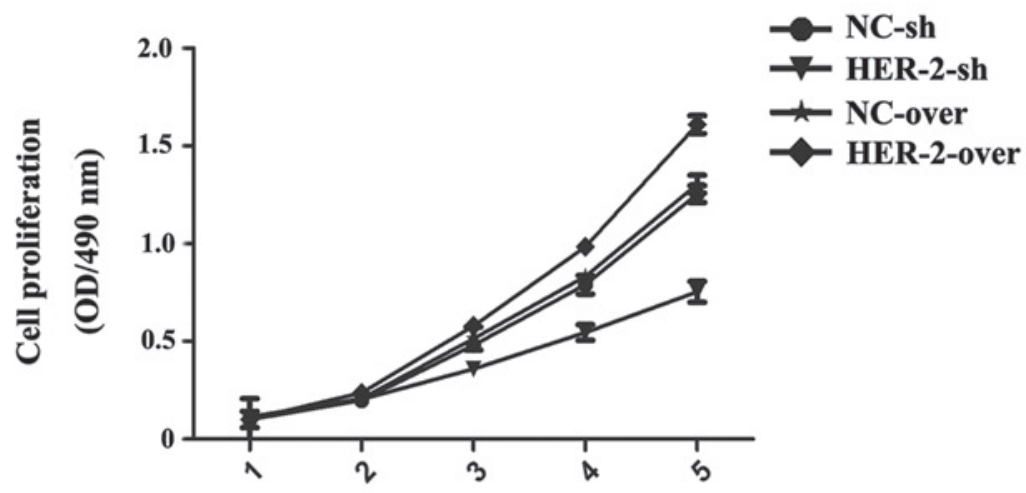

B

Time (days)

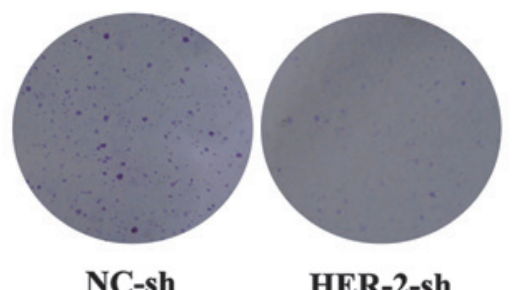

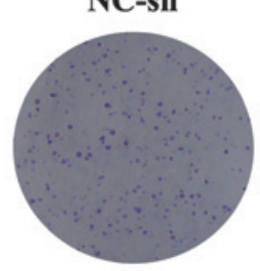

NC-over

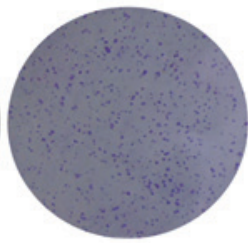

HER-2-over

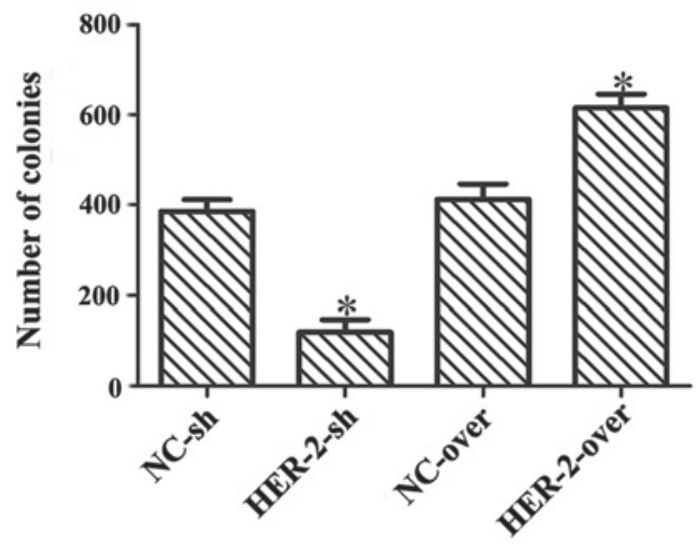

C
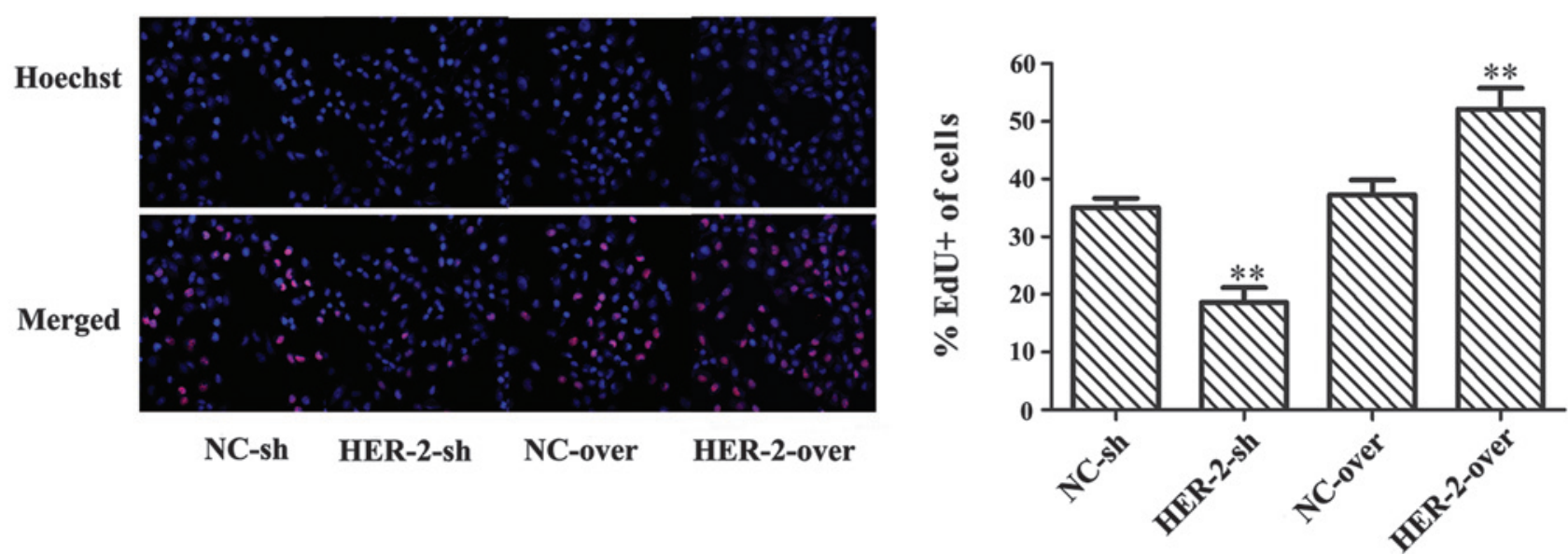

Figure 2. HER-2 increases cell proliferation, colony formation and DNA duplication in IOMM-Lee cells. (A) MTT assay revealed that, on the fifth day, HER-2-sh cell resistance was 1.66-fold higher than that of the NC-sh cells, whereas the resistance of the HER-2-over cells was reduced $24.4 \%$, compared with the NC-over cells $(\mathrm{P}<0.05)$. (B) In the colony formation assay, the number of colonies in the HER-2-sh cells decreased 69.1\%, compared with the NC-sh cells, whereas the number of colonies in the HER-2-over cells increased ("P<0.05). Magnification, $\mathrm{x} 1$. (C) In the EdU labeling assay, compared with the NC controls, positive labeling of the HER-2-sh cells was decreased and that of the HER-2-over cells was increased $\left.{ }^{* *} \mathrm{P}<0.05\right)$. Newly synthesized DNA is stained red by EdU and nuclei are stained blue by Hoechst 33342; magnification, x200. The data are expressed as the mean standard deviation from three independent experiments. $\mathrm{NC}$, negative control; sh, short hairpin; over, overexpression; OD, optical density.

$(\mathrm{P}<0.01)$. Western blot analysis also revealed similar effects on the protein levels of HER-2 $72 \mathrm{~h}$ post-infection (Fig. 1B), suggesting that the protein level of HER-2 in the HER-2-sh group decreased and that in the HER-2-overexpression group increased, compared with the mock or NC cells.
HER-2 increases cell proliferation in IOMM-Lee cells. The present study observed a significant decrease in proliferation following transfection with the silence lentiviral vector in the MTT assay. On the fifth day, the HER-2-sh cell resistance was 1.66-fold higher than that of the NC-sh cells $(\mathrm{P}<0.05$; Fig. 2A). 
A
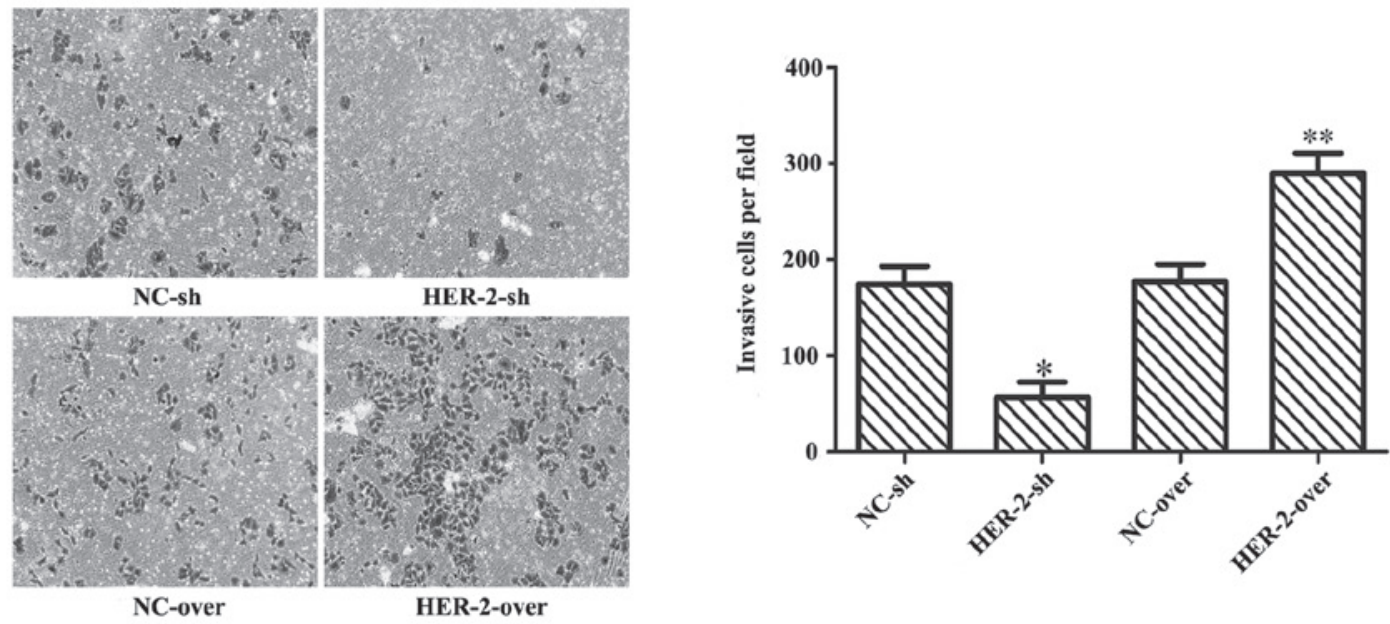

B

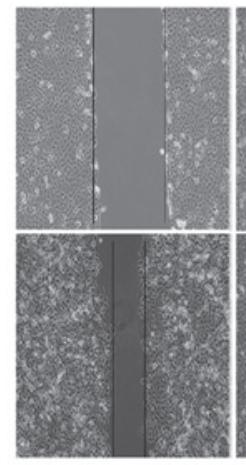

NC-sh

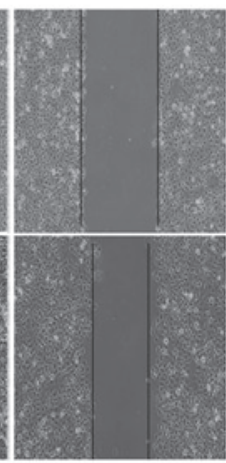

HER-2-sh

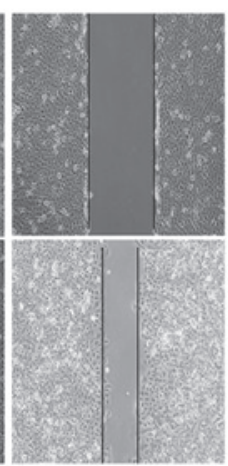

NC-over

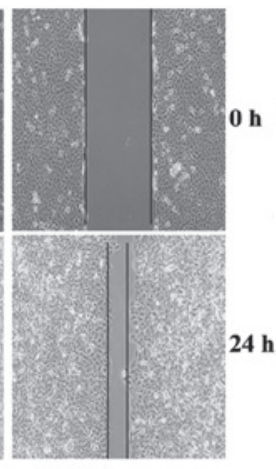

HER-2-over

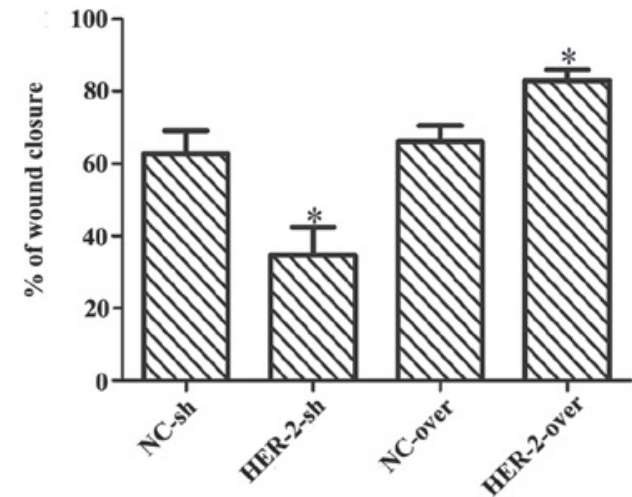

Figure 3. HER-2 increases cell invasion and migration in IOMM-Lee cells. IOMM-Lee cells were transfected with HER-2-sh, HER-2-over or NC control, the were then subjected to Transwell and wound healing assays. (A) Representative images of Transwell invasion assays, demonstrating that invasion of HER-2-sh was less than NC-sh and of HER-2-over was more than NC-over. (B) Respresentative images of the wound healing assay. The migration rate was observed to be lower in cells treated with HER-2-sh than that in NC-sh, and the migration rate was higher in cells treated with HER-2-over than that with NC-over. $\left({ }^{*} \mathrm{P}<0.05\right.$ and ${ }^{* *} \mathrm{P}<0.01$; independent $\mathrm{t}$-test). Magnification, $\mathrm{x} 200$. The data are expressed as the mean standard deviation from three independent experiments. $\mathrm{NC}$, negative control; sh, short hairpin; over, overexpression.

In the colony formation assay, the number of colonies in the HER-2-sh cell group decreased by $69.1 \%$, compared with that in the NC-sh cell group $(\mathrm{P}<0.05$; Fig. $2 \mathrm{~B})$. In the EdU labeling assay, a decrease in cell proliferation was observed in the HER-2-sh cells (1.88-fold), compared with the NC-sh cells $(\mathrm{P}<0.05$; Fig. 2C). By contrast, the overexpression lentiviral vector exhibited significantly increased cell proliferation in the MTT assay, and the results of the colony formation assay and EdU labeling assay were comparatively higher, at 24.4, 49 and $39.5 \%$, respectively $(\mathrm{P}<0.05$; Fig. $2 \mathrm{~A}-\mathrm{C})$. These results indicated that the proliferative ability of the IOMM-Lee cells was significantly enhanced by increasing the expression of HER-2.

Effect of HER-2 on IOMM-Lee cells invasion. To determine whether HER-2 affected the invasion and migration of IOMM-Lee cells, Matrigel invasion assays and wound healing assays were performed. The results suggested that increasing the expression of HER-2 enhanced the motility of the IOMM-Lee cells, while downregulation of HER-2 significantly inhibited the invasion and migration of the IOMM-Lee cells. As shown in Fig. 3A, compared with the NC cells, the invading ability of the HER-2-sh-transfected IOMM-Lee cells was significantly lower $(67.6 \%$; $\mathrm{P}<0.01)$, whereas the invasion of the HER-2-overexpression cells increased by $63.6 \%$ $(\mathrm{P}<0.05)$. The migrating ability of the HER-2-sh IOMM-Lee cells was significantly reduced by $44.7 \%$, compared with the $\mathrm{NC}$ cells, but was increased in the HER-2-overexpression cells by $25.8 \%(\mathrm{P}<0.05$; Fig. $3 \mathrm{~B})$.

Effect of HER-2 on IOMM-Lee cell cycle. The present study further investigated the effect of HER-2 on the cell cycle using flow cytometry. Downregulation of the HER-2 gene resulted in a significantly higher percentage of cells in the G1/G0 phase and decreased percentage of cells in the $S$ phase. By contrast, overexpression of HER-2 had the opposite effect $(\mathrm{P}<0.05$; Fig. 4A). Collectively, these data suggested that HER-2 enhanced the proliferation of the IOMM-Lee cells by promoting the G1-S cell cycle transition. The effect of HER-2 on cell apoptosis was further investigated and the results revealed that decreasing the expression of HER-2 increased apoptosis significantly. By contrast, overexpression of HER-2 decreased apoptosis of the IOMM-Lee cells $(\mathrm{P}<0.05$; Fig. 4B). 
A
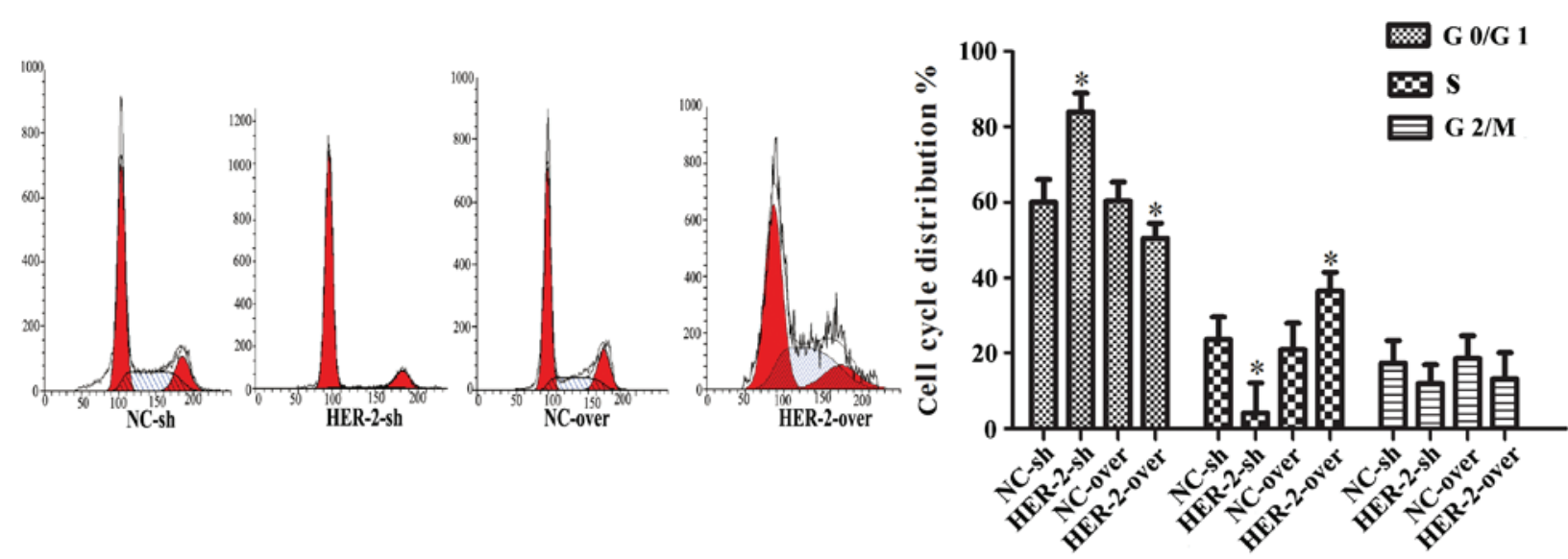

B
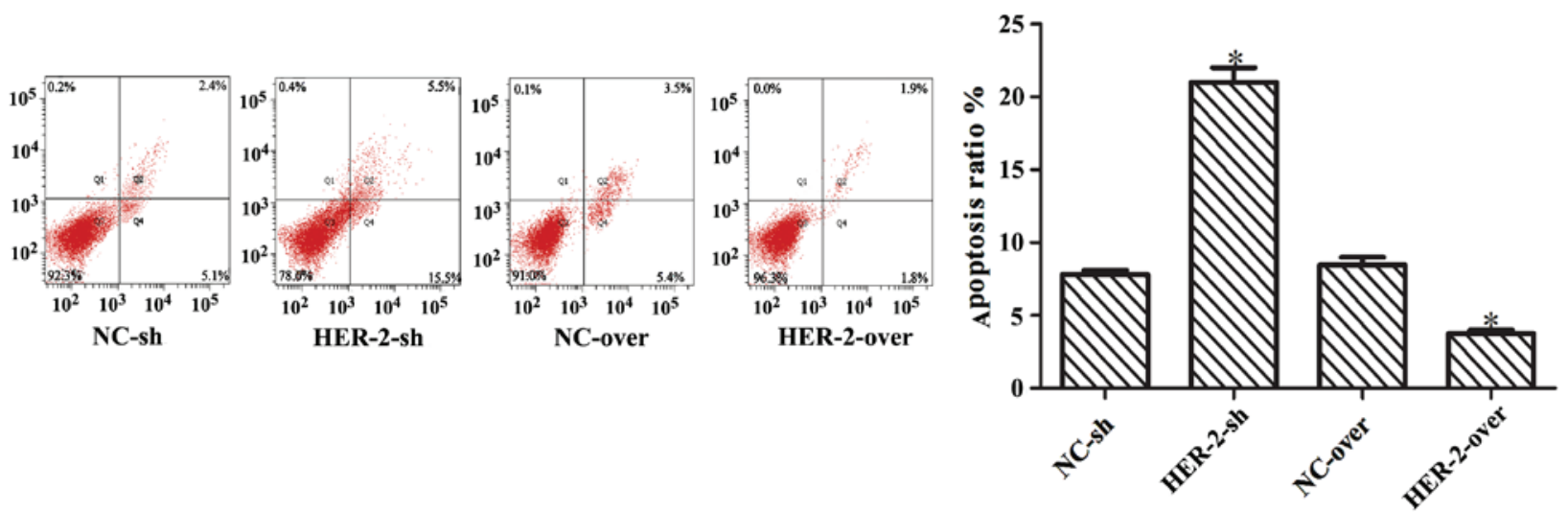

Figure 4. Effect of HER-2 on the cell cycle in IOMM-Lee cells. (A) Distribution of cells throughout the cell cycle were analyzed using flow cytometry ('P<0.0; independent t-test). (B) Effect of HER-2 on cell apoptosis. The cells undergoing apoptosis (early stage apoptosis, Q4; late stage apoptosis, Q2) were annexin-V-PE-positive and 7-AAD-negative ("P<0.05; independent t-test). The apoptotic ratio of HER-2-sh was increased compared with NC-sh, and HER-2-over was reduced compared with NC-over. The data are expressed as the mean standard deviation from three independent experiments. NC, negative control; sh, short hairpin; over, overexpression.

A

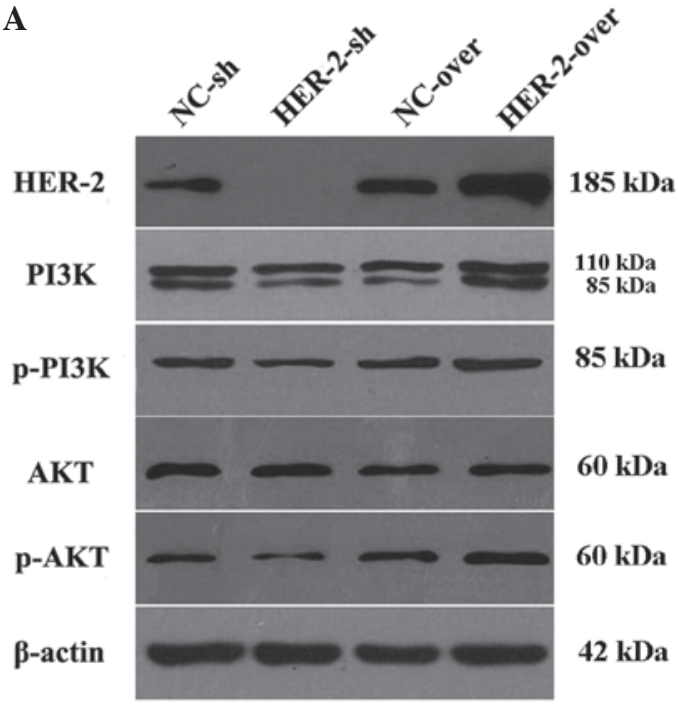

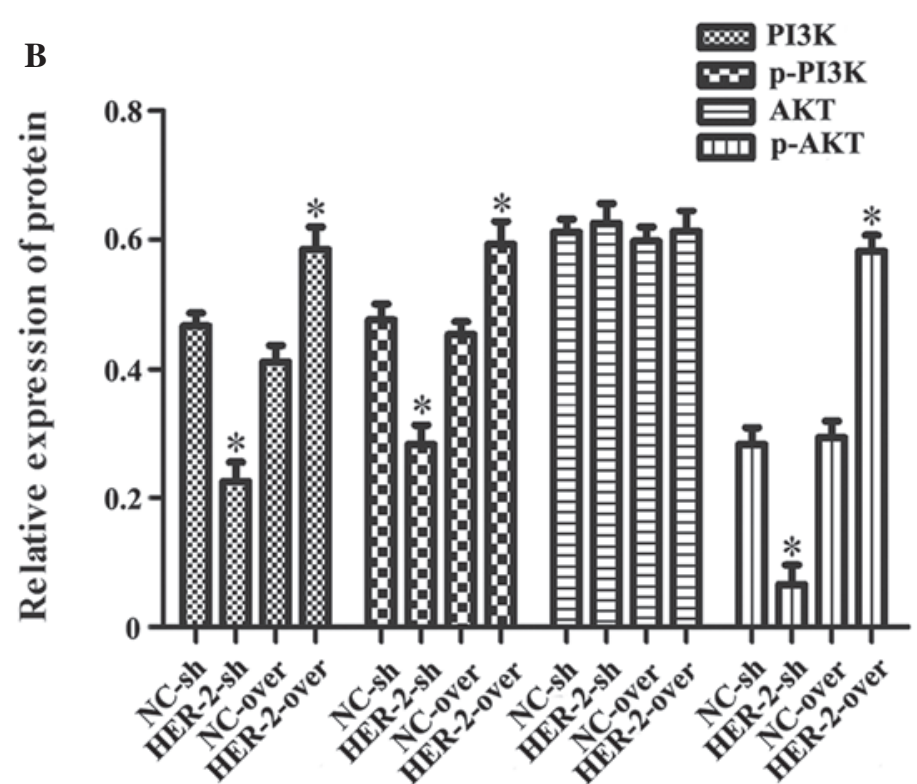

Figure 5. HER-2 affects the protein expression levels of PI3K and AKT in IOMM-Lee cells. (A) Protein levels of PI3K, p-PI3K, AKT and p-AKT in the IOMM-Lee cells were determined using western blot analysis $72 \mathrm{~h}$ post-transfection. $\beta$-actin was used as an internal loading control. (B) Protein levels of PI3K, p-PI3K and p-AKT in the HER-2-sh group were markedly decreased. In the HER-2-over group, the protein levels of PI3K, p-PI3K and p-AKT were increased, compared with the $\mathrm{NC}$ control and following normalization against $\beta$-actin $\left({ }^{*} \mathrm{P}<0.05\right)$. No difference was observed between the protein expression of AKT in the HER-2-sh and HER-2-over cells and that in the NC control was no difference. The data are expressed as the mean standard deviation from three independent experiments. NC, negative control; sh, short hairpin; over, overexpression; PI3K, phosphatidylinositol 3-kinase; p-, phosphorylated. 
HER-2 affects protein expression levels of PI3K and AKT in IOMM-Lee cells. In order to investigate the association between HER-2 and the activity of the PI3K/AKT signaling pathway, the present study measured the protein levels of PI3K, p-PI3K, AKT and p-AKT in the IOMM-Lee cells following transfection. The results revealed that, compared with NC group, the protein levels of PI3K, p-PI3K and p-AKT in the downregulated HER-2 group were significantly reduced by 51.6, 40.5 and $76 \%$, respectively $(\mathrm{P}<0.05$; Fig. 5A and $\mathrm{B})$. By contrast, in the upregulated HER-2 cells, the protein levels of PI3K, p-PI3K and p-AKT increased by 41.9, 30.6 and $99.4 \%$, respectively $(\mathrm{P}<0.05$; Fig. $5 \mathrm{~A}$ and $\mathrm{B})$. However, no difference was observed in the protein expression of AKT in these two groups, compared with the NC group.

\section{Discussion}

HER-2 is an oncogene in human carcinoma, and a number studies have demonstrated that it is significantly upregulated in several types of human malignancy, with studies indicating that HER-2 is important in tumor cell proliferation, invasion and apoptosis $(22,23)$. Our previous study demonstrated that overexpression of HER-2 in patients with meningioma was associated with poor prognosis (24). The exact nature of the contribution made by increased protein levels of HER-2 to human meningioma cell proliferation and motility during the progression of carcinoma, however, remains to be fully elucidated.

In the present study, HER-2 was demonstrated to regulate the biochemical characteristics of human malignant meningioma cells. When the gene expression of HER-2 was downregulated, the proliferative ability of the cells declined, determined using MTT, colony formation and Edu labeling assays, and the invasive ability was decreased, observed using a Matrigel invasion assay. In terms of the cell cycle, these cells were arrested at the G0/G1-phase and apoptosis was increased. When the gene expresion of HER-2 was upregulated, the proliferate ability of the cells increased, and the invasive and migration abilities increased significantly. The cell cycle was promoted at the G1/S-phase and apoptosis was decreased. Therefore, the present study demonstrated that HER-2 promoted cell proliferation and invasion and inhibited apoptosis in the human malignant meningioma IOMM-Lee cells.

It has been reported that the oncogenic effects of HER-2 depend predominantly on preservation of the 'lipogenic phenotype' (25). Ligand stimulation induces dimerization of the HER receptor, either to a homodimer or heterodimer, which leads to self-phosphorylation on tyrosine residues localized to the C-terminal domain of the HER receptors (26). HER-2 is the preferred heterodimerization partner of all EGFR proteins, and is important in the lateral transmission of signals between other EGFR receptors (7). The activated phosphorylated HER receptors then activate a variety of downstream signaling modules, includeing the PI3K/Akt/mammalian target of rapamycin (mTOR) pathway, the mitogen-activated protein kinase pathway and the phospholipase C pathway (27).

The PI3K/AKT pathway is an essential pathway for various cellular processes, including cell growth, cell survival, motility, angiogenesis and metabolism $(28,29)$. Therefore, the present study hypothesized that HER-2 can affect the protein synthesis or activities of PI3K/AKT, leading to the facilitation of cell motility and invasion. To assess this hypothesis, the present study used western blot analysis to detect the expression levels of PI3K/AKT. Upregulation of the expression of HER-2 led to increased levels of PI3K. PI3K is pivotal in further signaling of the pathway, as it is the substrate of various protein kinases containing a pleckstrin homology domain, including the serine-threonine kinase AKT (30). AKT kinases belong to the AGC kinase family, associated with AMP/GMP kinases and protein kinase C (31). Activated AKT can phosphorylate a number of downstream effectors, regulating a variety of essential processes, including protein synthesis, cell metabolism, cell growth/proliferation, cell survival and resistance to various exogenous stresses (32). Full AKT activation depends on the concomitant phosphorylation of two distinct sites, which can be activated independently. PDK-1-catalyzed T308 phosphorylation inside the activation loop serves as a readout of PI3K activation. By contrast, phosphorylation of S473 in the hydrophobic motif of the C-terminal tail indicates mTORC2 to AKT feedback signaling activity or induction by the PI3K-related kinase superfamily or DNA-dependent protein kinase (33). There are also other signals, which regulate AKT, including the extracellular signal-regulated kinase (ERK)/MAPK signaling pathway and the Janus kinase-signal transducers and activators of transctition pathway $(34,35)$. Therefore, in the present study, the levels of p-AKT increased with the increase in p-PI3K, however, AKT may not be changed completely. Further investigations are required to clarify the exact molecular mechanisms.

In conclusion, the results of the present study indicated that overexpression of HER-2 stimulated human meningioma cell proliferation and invasion, which may contribute to meningioma development and progression. These results may explain, in part, the observation that the overexpression of HER-2 during the progression of meningioma is clinically associated with a high metastatic potential and poor prognosis. Furthermore, variation in the expression of HER-2 affected the protein levels of PI3K and AKT in the IOMM-Lee cells, which provided evidence for a functional linkage between HER-2 signaling and the activity of PI3K/AKT in cell invasion. These results suggested that the HER-2/PI3K/AKT pathway may be a valuable target for the development of novel therapeutic approaches to prevent or suppress cell invasion and metastasis in meningioma.

\section{Acknowledgements}

This study was supported by grants from the National Natural Science Foundation of China (grant. no. 81260372) and the Natural Science Foundation of Jiangxi Province (grant. no. 20114BAB205047).

\section{References}

1. Louis DN, Ohgaki H, Wiestler OD, et al: The 2007 WHO classification of tumours of the central nervous system. Acta Neuropathol 114: 97-109, 2007.

2. Chen L, Zou X, Wang Y, Mao Y and Zhou L: Central nervous system tumors: a single center pathology review of 34, 140 cases over 60 years. BMC Clin Pathol 13: 14, 2013. 
3. Mawrin C and Perry A: Pathological classification and molecular genetics of meningiomas. J Neurooncol 99: 379-391, 2010.

4. Andrae N, Kirches E, Hartig R, et al: Sunitinib targets PDGF-receptor and Flt3 and reduces survival and migration of human meningioma cells. Eur J Cancer 48: 1831-1841, 2012.

5. Vranic A: Antigen expression on recurrent meningioma cells. Radiology and oncology 44: 107-112, 2010.

6. Sahlberg KK, Hongisto V, Edgren $\mathrm{H}$, et al: The HER2 amplicon includes several genes required for the growth and survival of HER 2 positive breast cancer cells. Mol Oncol 7: 392-401, 2013

7. Liu X, Zhang Y, Ren W and Rao G: ErbB2 gene silencing and its effect on PTEN in SACC-83 salivary adenoid cystic carcinoma cells. Oncol Rep 24: 1291-1296, 2010.

8. Way TD, Kao MC and Lin JK: Apigenin induces apoptosis through proteasomal degradation of HER $2 / n e u$ in HER2/neu-overexpressing breast cancer cells via the phosphatidylinositol 3-kinase/Akt-dependent pathway. J Biol Chem 279: 4479-4489, 2004.

9. Park HK, Kim IH, Kim J and Nam TJ: Induction of apoptosis and the regulation of ErbB signaling by laminarin in HT-29 human colon cancer cells. Int J Mol Med 32: 291-295, 2013.

10. Paik S, Bryant J, Tan-Chiu E, et al: HER 2 and choice of adjuvant chemotherapy for invasive breast cancer: National Surgical Adjuvant Breast and Bowel Project Protocol B-15. J Natl Cancer Inst 92: 1991-1998, 2000.

11. Pegram MD: Treating the HER 2 pathway in early and advanced breast cancer. Hematol Oncol Clin North Am 27: 751-765, 2013

12. Di Cosimo S, Arpino G and Generali D: Neoadjuvant treatment of HER2 and hormone-receptor positive breast cancer-moving beyond pathological complete response. Breast 23: 188-192, 2014.

13. Ding H, Helguera G, Rodriguez JA, et al: Polymalic acid nanobioconjugate for simultaneous immunostimulation and inhibition of tumor growth in HER2/neu-positive breast cancer. J Contro Release 171: 322-329, 2013.

14. Boku N: HER2-positive gastric cancer. Gastric Cancer 17: 1-12, 2014.

15. Qi W, Li X, Zhang Y, et al: Overexpression of Her-2 upregulates FoxM1 in gastric cancer. Int J Mol Med 33: 1531-1538, 2014

16. Jeung J, Patel R, Vila L, et al: Quantitation of HER $2 /$ neu expression in primary gastroesophageal adenocarcinomas using conventional light microscopy and quantitative image analysis. Arch Pathol Lab Med 136: 610-617, 2012.

17. Chao WR, Lee MY, Lin WL, et al: HER2 amplification and overexpression are significantly correlated in mucinous epithelial ovarian cancer. Hum Pathol 45: 810-816, 2014.

18. Waage IS, Vreim I and Torp SH: C-erbB2/HER2 in Human Gliomas, Medulloblastomas and Meningiomas: A Minireview. Int J Surg Pathol 21: 573-582, 2013.

19. Mahzouni P and Movahedipour M: An immunohistochemical study of HER 2 expression in meningioma and its correlation with tumor grade. Pathol Res Pract 208: 221-224, 2012.
20. Loussouarn D, Brunon J, Avet-Loiseau H, et al: Prognostic value of HER2 expression in meningiomas: an immunohistochemical and fluorescence in situ hybridization study. Hum Pathol 37: 415-421, 2006

21. Abdelzaher E,El-Gendi SM, Yehya A and Gowil AG: Recurrence of benign meningiomas: predictive value of proliferative index, BCL2, p53, hormonal receptors and HER2 expression. $\mathrm{Br}$ J Neurosurg 25: 707-713, 2011.

22. Fu J, Tian C, Xing M, et al: KU004 induces G1 cell cycle arrest in human breast cancer SKBR-3 cells by modulating PI3K/Akt pathway. Biomed Pharmacother 68: 625-630, 2014.

23. Khan S, Shukla S, Sinha S, Lakra AD, Bora HK and Meeran SM: Centchroman suppresses breast cancer metastasis by reversing epithelial-mesenchymal transition via downregulation of HER2/ERK1/2/MMP-9 signaling. Int J Biochem Cell Biol 58: $1-16,2015$

24. Wang CL, Mei JH, Wang SS, et al: Expression of HER2/neu in meningiomas: an immunohistochemistry and fluorescence in situ hybridization study. Zhonghua Bing Li Xue Za Zhi 39: 156-160, 2010 (In Chinese).

25. Lin VC, Chou CH, Lin YC, et al: Osthole suppresses fatty acid synthase expression in HER2-overexpressing breast cancer cells through modulating Akt/mTOR pathway. J Agr Food Chem 58: 4786-4793, 2010

26. Kuo HP, Hsu SC, Ou CC, et al: Ganoderma tsugae extract inhibits growth of HER2-overexpressing cancer cells via modulation of HER2/PI3K/Akt signaling pathway. Evid Based Complement Alternat Med 2013: 219472, 2013.

27. Roskoski R Jr: The ErbB/HER family of protein-tyrosine kinases and cancer. Pharmacol Res 79: 34-74, 2014

28. Courtney KD, Corcoran RB and Engelman JA: The PI3K pathway as drug target in human cancer. J Clin Oncol 28: 1075-1083, 2010.

29. Bartholomeusz C and Gonzalez-Angulo AM: Targeting the PI3K signaling pathway in cancer therapy. Expert Opin Ther Tar 16: 121-130, 2012.

30. Steelman LS, Stadelman KM, Chappell WH, et al: Akt as a therapeutic target in cancer. Expert Opin Ther Targets 12: 1139-1165, 2008

31. Porta C, Paglino C and Mosca A: Targeting PI3K/Akt/mTOR signaling in cancer. Front Oncol 4: 64, 2014.

32. Lauring J, Park BH and Wolff AC: The Phosphoinositide-3-kina se-Akt-mTOR pathway as a therapeutic target in breast cancer. J Natl Compr Canc Netw 11: 670-678, 2013.

33. Briest F and Grabowski P: PI3K-AKT-mTOR-signaling and beyond: the complex network in gastroenteropancreatic neuroendocrine neoplasms. Theranostics 4: 336-365, 2014.

34. Jang YN and Baik EJ: JAK-STAT pathway and myogenic differentiation. Jak-Stat 2: e23282, 2013

35. Kavitha K, Kowshik J, Kishore TK, et al: Astaxanthin inhibits NF-kappaB and Wnt/beta-catenin signaling pathways via inactivation of Erk/MAPK and PI3K/Akt to induce intrinsic apoptosis in a hamster model of oral cancer. Biochim Biophys Acta 1830: 4433-4444, 2013. 